\title{
The Prevalence of Thyroid Papillary Microcarcinoma in Patients With Benign Thyroid Fine Needle Aspiration
}

\author{
Abdullah H. Alshathry ${ }^{1}$, Nawaf Z. Almeshari ${ }^{1}$, Abdulaziz S. Alarifi ${ }^{1}$, Abdullah M. Aleidy ${ }^{1}$, Saleh Aldhahri \\ 2 \\ 1. Otolaryngology, College of Medicine, King Saud University, Riyadh, SAU 2. Otolaryngology, King Saud University, \\ Riyadh, SAU
}

Corresponding author: Saleh Aldhahri, saldhahri@ksu.edu.sa

\section{Abstract}

\section{Introduction}

Thyroid nodules are a very common clinical finding in the general population. We use fine needle aspiration (FNA) as the gold standard workup test for a thyroid nodule, as it is capable of differentiating malignant nodules from benign in the majority of cases. Usually, FNA is done for nodules that are more than $1 \mathrm{~cm}$; small malignant lesions that are less than $1 \mathrm{~cm}$ in size can be missed. That's why the risk of having undiagnosed microcarcinomas in an otherwise benign FNA needs to be explored.

\section{Aim}

To estimate the prevalence of thyroid papillary microcarcinoma in patients with benign FNA and evaluate and correlate the FNA cytological results with the final histopathological diagnoses.

\section{Methods}

This was a retrospective study of 1543 post-thyroidectomy patients who underwent FNA cytology, were classified according to the Bethesda scoring system, and were admitted to two tertiary care hospitals in Riyadh, Saudi Arabia, from 2010 to 2019.

\section{Results}

Six-hundred-seven (607) out of 1543 FNA cytology results were reported as benign, 215 as malignant, and 73 as suspicious of malignancy. On final histopathology diagnosis, 81/607 (13.34\%) of benign cases and 35/215 (16.28\%) of malignant cases did not meet the initial cytology and were confirmed as papillary microcarcinoma. In patients with microcarcinoma after initial benign FNA (89.2\%) found to have benign multinodular changes, compared to only (31\%) of initial malignant FNA patients.

Review began $11 / 11 / 2020$ Review ended 11/20/2020 Published 12/01/2020

\section{() Copyright 2020}

Alshathry et al. This is an open access article distributed under the terms of the Creative Commons Attribution License CC-BY 4.0., which permits unrestricted use, distribution, and reproduction in any medium, provided the original author and source are credited.

\section{Conclusion}

When non-surgical intervention is chosen in patients with benign FNA, the possibility of coexisting microcarcinoma with its variable prognosis should be taken into account and explained to the patient.

Categories: Otolaryngology, General Surgery

Keywords: thyroid, microcarcinoma, ptc, fna, bethesda

\section{Introduction}

Thyroid nodules are a very common finding in clinical practice with a prevalence reported between $4 \%$ and $7 \%$ in the general population [1-2]. The ultimate goal when evaluating a patient with a thyroid nodule is to differentiate between benign and malignant lesions or at least to estimate the risk of malignancy in the existing nodule. According to the National Institute of Health $(\mathrm{NIH})$, around $1.3 \%$ of the general population at some point in their lives will be diagnosed with thyroid gland cancer [3]. This high percentage emphasizes the need for the appropriate utilization and proper interpretation of the available diagnostic tests to achieve accurate and early diagnosis and to have the best possible outcome. Since its introduction to the medical field, fine needle aspiration (FNA) has proven itself to be the mainstay tool and the method of choice for the diagnosis of thyroid nodules [4]. FNA's accuracy as a diagnostic tool combined with its simplicity, costeffectiveness, and safe usage has made it the predominant tool for us. This simple diagnostic test complemented by other clinical and radiological findings guides the management of thyroid nodules, as it provides the necessary information regarding which patients will require surgical intervention from those who will not [5]. In terms of reporting the FNA results, Bethesda scoring system has been set as a standard way to classify the results according to cytopathologic features and the risk of malignancy: (1-Non diagnostic, 2-Benign, 3-Atypia/Follicular Lesion with Atypia of Undetermined Significance, 4-Follicular 


\section{Cureus}

Neoplasm or Suspicious for a Follicular Neoplasm, 5- Suspicious of Malignancy, 6-Malignant) [6]. Because a thyroid FNA is usually done for the most suspicious nodule (based on the size and radiological features) and because the FNA inherited diagnostic limitation when done for nodules that are smaller than $1 \mathrm{~cm}$, small malignant lesions that are less than $1 \mathrm{~cm}$ in size can be missed and only discovered after the surgical removal of the thyroid [7]. So, for better patient counseling, the risk of having undiagnosed microcarcinomas in an otherwise benign FNA needs to be explored.

\section{Materials And Methods}

A retrospective cohort study was conducted in two tertiary care hospitals in Riyadh, Saudi Arabia. Patients who underwent thyroid surgery with preoperative FNA between 2010 to 2019 were included. Patients who refused to do FNA and those who underwent the surgery without preoperative FNA were excluded from the study. There were 1543 thyroid surgeries performed with preoperative FNA during this period. All surgeries were done by head and neck consultants with more than 10 years of experience in thyroid surgery. The patient's FNA cytology was categorized according to the Bethesda reporting system into six categories (1Non-diagnostic, 2-Benign, 3-Atypia/Follicular Lesion with Atypia of Undetermined significance, 4-Follicular Neoplasm or Suspicious for a Follicular Neoplasm, 5-Suspicious of Malignancy, 6-Malignant) [6]. The final histopathology was classified as "Benign," "Papillary Microcarcinoma" if the largest malignant focus measures $1 \mathrm{~cm}$ or less, or "Malignant" for papillary carcinoma that measures more than $1 \mathrm{~cm}$ in the largest diameter or other thyroid malignancies [8].

The patient's medical data were collected using electronic charts in both hospitals. Demographic parameters, such as gender, body mass index (BMI), pre-operative thyroid-stimulating hormone (TSH), antithyroglobulin antibody, and anti-thyroid peroxidase antibody were evaluated. Data were entered and analyzed by using Stata program vision 15 (StataCorp LLC, College Station, Texas), simple descriptive statistics used to generate the mean, standard deviation, frequencies, and percentages. When relevant, the independent $\mathrm{t}$-test and the chi-square test used to determine any statistical significance for continuous and nominal variables respectively. Anonymity and confidentiality were maintained in all phases of the study. This study has been approved by the institutional review board, research ethics committee in King Fahad Medical City.

\section{Results}

The study included 1543 patients with 1242 females (80.49\%) and 301 males (19.51\%), with a mean age of 43.07 years for males and 40.6 for females. The BMI varies among the study group with a mean of 29.4 for males and 31.03 for females. Preoperative TSH and vitamin D values show no statistically significant difference between males and females $(\mathrm{p}=0.80)$ and $(\mathrm{p}=0.51)$, respectively. Out of a total of 830 patients, $38 \%$ had a positive preoperative anti-thyroglobulin antibody test; $84.2 \%$ of them were female with a p-value of 0.008 . Moreover, 823 patients had done the anti-thyroid peroxidase antibody test, $29 \%$ were found to be positive, with $86.2 \%$ of them being female with a p-value of 0.005 (Table 1 ).

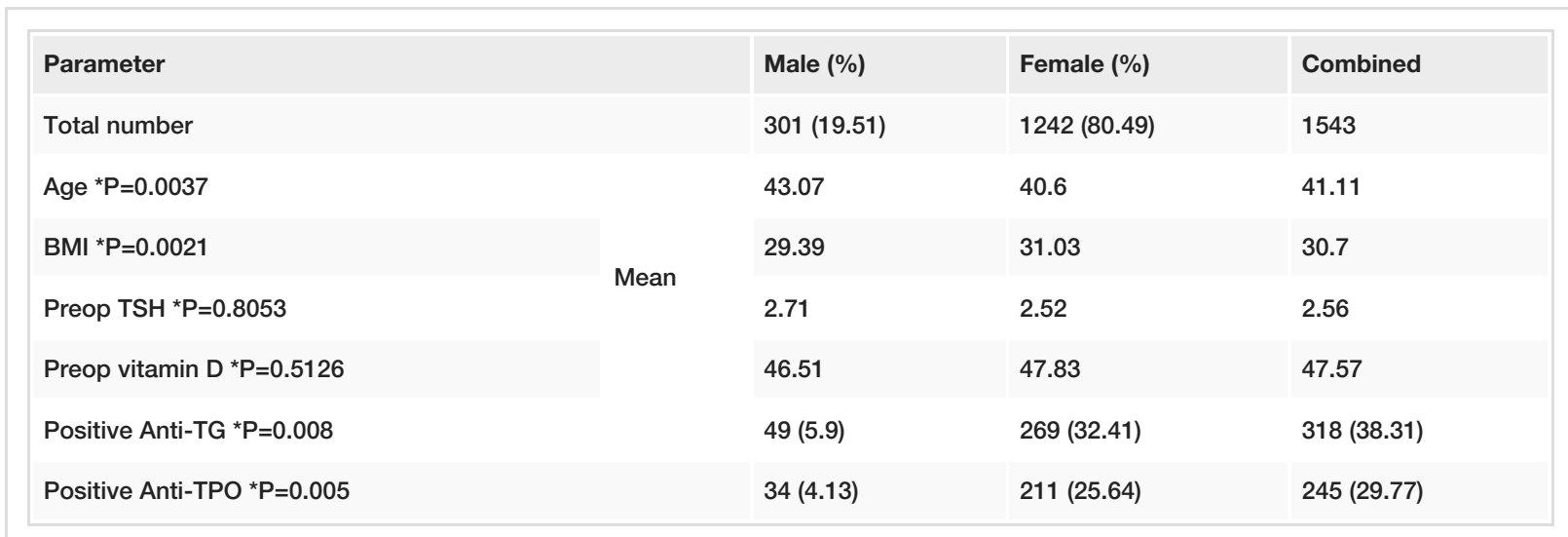

\section{TABLE 1: Demographic data}

BMI (body mass index), anti-TG (anti-thyroglobulin antibody test), anti-TPO (anti-thyroid peroxidase antibody), preop (preoperative), P (p-value)

The thyroid FNA results were categorized based on the Bethesda scoring system into six categories as follows (Figure 1). 


\section{Cureus}

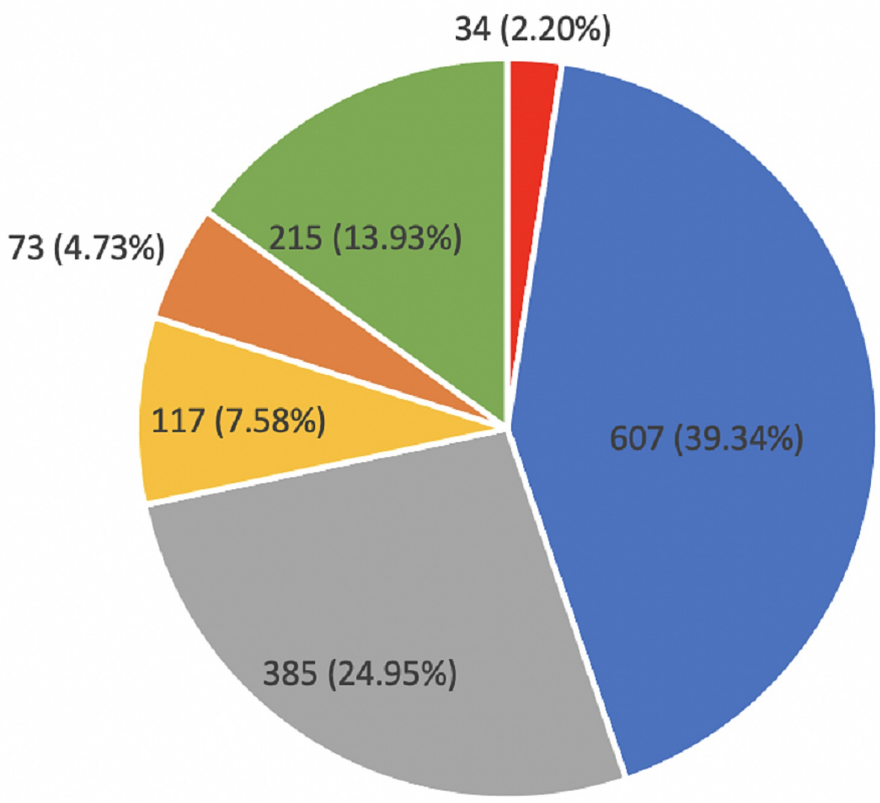

- Non-diagnostic (category 1)

- Benign (category 2)

- Atypia/Follicular lesion of Undetermined significance (category 3)

घ Follicular Neoplasm or Suspicious for a Follicular Neoplasm (category 4)

- Suspicious of Malignancy (category 5)

- Malignant (category 6)

\section{FIGURE 1: Distribution of FNA results based on the Bethesda scoring system}

FNA (fine needle aspiration)

The final histopathologic diagnosis showed 823 patients with benign pathology, 470 patients with malignant pathology, and 250 patients with papillary microcarcinoma. Out of 607 patients with initially benign FNA, $76(12.52 \%)$ final histopathologic examination came back as malignant tumor and $81(13.34 \%)$ patients were diagnosed with papillary microcarcinoma. On the other hand, out of 215 patients with initially malignant FNA, the final pathology of 175 (81.40\%) matched their initial result and 35 cases (16.28\%) were diagnosed as microcarcinoma. The malignancy rates in the remaining categories can be found in Table 2. More details regarding the final pathology can be found in Table 3 . 


\section{Cureus}

\begin{tabular}{|c|c|c|c|c|}
\hline \multirow[b]{2}{*}{ FNA code } & \multicolumn{3}{|l|}{ Final Pathology } & \multirow[b]{2}{*}{ Total } \\
\hline & Benign & Malignant & Microcarcinoma & \\
\hline 0 & $66(58.93 \%)$ & $29(25.89 \%)$ & 17 (15.18\%) & 112 (100\%) \\
\hline 1 & $25(73.53 \%)$ & $7(20.59 \%)$ & $2(5.88 \%)$ & $34(100 \%)$ \\
\hline 2 & 450 (74.14\%) & $76(12.52 \%)$ & 81 (13.84\%) & 607 (100\%) \\
\hline 3 & 214 (55.58\%) & $96(24.94 \%)$ & 75 (19.48\%) & 385 (100\%) \\
\hline 4 & $54(46.15 \%)$ & $41(35.04 \%)$ & 22 (18.80\%) & 117 (100\%) \\
\hline 5 & 9 (12.33\%) & $46(63.01 \%)$ & 18 (24.66\%) & 73 (100\%) \\
\hline 6 & $5(2.33 \%)$ & $175(81.40 \%)$ & $35(16.28 \%)$ & $215(100 \%)$ \\
\hline lotal & $823(53.34 \%)$ & $4 / 0(30.46 \%)$ & $250(16.20 \%)$ & $1543(100 \%)$ \\
\hline
\end{tabular}

\section{TABLE 2: Thyroid FNA and final pathology outcome.}

FNA code: 0 (not done), 1 (non-diagnostics), 2 (benign), 3 (atypia/follicular lesion of undetermined significance), 4 (follicular neoplasm or suspicious for a follicular neoplasm), 5 (suspicious of malignancy), 6 (malignant)

FNA (fine needle aspiration)

\begin{tabular}{|c|c|c|}
\hline Pathology & Number of cases & $\%$ \\
\hline Benign pathology & 783 & 50.75 \\
\hline PTC & 402 & 26.16 \\
\hline Micro PTC & 250 & 16.07 \\
\hline FTC & 26 & 1.69 \\
\hline NIFTP & 22 & 1.43 \\
\hline Follicular lesion of uncertain malignant potential & 18 & 1.17 \\
\hline Minimally Invasive FTC / Hurthle cell Ca minimally invasive & 16 & 1.04 \\
\hline Multiple types of thyroid Ca & 6 & 0.38 \\
\hline Hurthle cell Ca & 5 & 0.32 \\
\hline Medullary thyroid $\mathrm{Ca}$ & 5 & 0.32 \\
\hline Anaplastic thyroid Ca & 4 & 0.26 \\
\hline Lymphoma & 4 & 0.26 \\
\hline Others & 2 & 0.12 \\
\hline Total & 1,543 & 100.00 \\
\hline
\end{tabular}

\section{TABLE 3: Final pathology}

PTC (papillary thyroid carcinoma), FTC (follicular thyroid carcinoma), NIFTP (non-invasive follicular thyroid neoplasm with papillary-like nuclear features), Cancer (Ca)

In patients with benign FNA who had a microcarcinoma, $89.2 \%$ of them have benign multinodular changes in the thyroid histopathology in comparison to only $31 \%$ in patients with malignant FNA who turned out to have only a microcarcinoma in the final pathology.

Seven-hundred twenty $(720 ; 46.66 \%)$ patients turned out to have a malignant tumor in the final pathology; 
papillary thyroid carcinoma was the most common malignant tumor (55.83\%), followed by papillary microcarcinoma (34.72\%). The others are listed in Table 3.

When evaluating the disease multicentricity in patients with benign FNA, there was no significant difference between patients with microcarcinoma and patients with a larger malignant lesion ( $31.7 \% \mathrm{vs}$ $36.7 \%$, respectively, with $\mathrm{P}=0.45$ ).

Considering a microcarcinoma as an incidental finding in the final pathology (because performing FNA for less than $1 \mathrm{~cm}$ nodules is not generally recommended) [7], the sensitivity and specificity of FNA in detecting malignancy in thyroid nodules (considering only Bethesda scores 2 and 6) are $69.72 \%$ and $92.99 \%$, respectively. The false-positive rate was $7 \%$ and the false-negative rate was $30.27 \%$. The positive predictive value was $81.39 \%$ and the negative predictive value was $87.47 \%$. The accuracy of FNA in differentiating benign from malignant thyroid lesions was $85.88 \%$.

After we correlated the results of the FNA and the final histopathological examination based on gender, we found that $9.80 \%$ of male patients with an initially benign FNA were diagnosed as malignant with $13.86 \%$ of females with benign initial FNA turning out to have a malignant tumor. However, using only the 2nd and 6th Bethesda categories, the sensitivity was higher in the male group (83.33\%) than in the female (64.10\%) while specificity, positive predictive value, and negative predictive value showed almost no difference between both genders.

\section{Discussion}

In 1960, FNA was introduced in Sweden as the initial diagnostic test to categorize thyroid nodules due to its simplicity and cost-effectiveness [9]. However, there have been some variations in the diagnostic yields of FNA from one institution to another. Several factors can be considered as a reason for this variation such as the experience of the technologist, whether it was done under ultrasound guidance or not, the accuracy of interpretation of the final cytological results, including the classification of suspicious lesions, and the reporting system of the thyroid cytology [10-11].

As expected, our series were predominantly female (female to male; 4:1) with a comparable mean of age, which is in keeping with other studies [12]. Moreover, other parameters, including (BMI, Vit D, pre-op TSH, anti-thyroglobulin antibody, and anti-thyroid peroxidase antibody) showed almost no significant differences between both genders' groups.

In our study, 39.34\% had an initially benign FNA, which is near what was reported by Lew et al. (32\% out of 797 [13]. However other studies have shown large variability in the rate of benign FNA, 59\% by Machala et al. and $14 \%$ by Wu et al. $[12,14]$. On the other hand, malignant FNA and those suspicious for malignancy cases in our study represent $18.66 \%$, similar to a study conducted in Poland with $15.2 \%$ out of 1262 cases reported to be malignant [12]. However, different percentages of malignant FNA were found in different publications where they were reported to be as low as $5.3 \%$ and $6.8 \%[14,15]$. This variability in the benign and malignant FNA rate can be attributed to the difference between the studied population. It did not surprise us that papillary thyroid carcinoma (PTC) and micro PTC were the most common malignant tumors, as that was in keeping with other studies [16-17].

Currently, thyroid cancer is considered the fastest growing cancer in the world, mostly due to the increasing number of incidental findings of papillary microcarcinoma in an otherwise benign thyroid [18]. Papillary thyroid microcarcinoma is defined by the World Health Organization (WHO) as a subtype of papillary thyroid cancer measuring $1 \mathrm{~cm}$ or less in size on the largest dimension [8]. Despite this increasing incidence, American Thyroid Association guidelines recommend not to do thyroid FNA in nodules less than $1 \mathrm{~cm}$ if they have a very low suspicion ultrasound (US) pattern [7].

Recently, different studies showed an increasing prevalence of microcarcinoma. In our series, almost onethird of the cases of thyroid cancers were confirmed as micro PTC. A meta-analysis conducted in 2008 showed that an average of $22.9 \%$ of malignant tumors are micro PTC [19]. Although more recent studies, such as the one reported by Louise Davies et al., showed a more comparable rate with ours (39\%) [20].

These percentages of papillary microcarcinoma are considered high, especially if they can be found in good numbers in patients with benign cytology, as in our study (13.34\%). The main reason for missing these malignant changes in benign FNA is the fact that the biopsy usually directs toward the largest/most suspicious nodule in US that is more than $1 \mathrm{~cm}$ in size according to the guidelines [7]. Moreover, in $59.6 \%$ of our patients with microcarcinoma, their nodule size was $5 \mathrm{~mm}$ or less, which makes it even more difficult to biopsy preoperatively. Several other factors could be responsible; one of these could be the presence of benign multinodular changes in almost one-third of patients with benign FNA with micro PTC compared to only $4 \%$ to those with larger than $1 \mathrm{~cm}$ malignant tumor. Having only one focus of micro PTC is another factor that may make the preoperative diagnosis even more difficult. Of the micro PTC, $67.13 \%$ has only a single focus in a multinodular goiter. 
Although the prognosis for papillary microcarcinoma is better than other, large, well-differentiated thyroid cancer [21-22], we still do not know whether this better outcome is related to the nature of the disease or it is the early discovery. In general, unifocal micro PTC carries an excellent prognosis, however, multifocality, size above $6 \mathrm{~mm}$, and age have been recognized as some prognostic factors that can alter this excellent outcome. Unfortunately, due to the excellent outcome of thyroid cancers in general, and the long follow-up needed to assess the impact of any intervention on prognosis, it is extremely difficult to do clinical trials assessing the outcome based on the intervention [23-25].

The reported sensitivity and specificity of thyroid FNA were $69.72 \%$ and $92.99 \%$, respectively. This shows that the FNA has a moderate satisfactory ability in detecting thyroid malignancy in our study. However, many studies in the literature show values of sensitivity as low as $55.3 \%, 60.28 \%$, and $61.53 \%[10,12,26]$. According to the literature, the causes of this low sensitivity may be attributed to the use of US guidance, variability in the operator techniques and expertise, and the classification of undetermined and suspicious lesions [27-28]. On the other hand, higher ranges of sensitivity have been reported with $82.14 \%$ and $89.5 \%$, considering that both of these studies had a smaller sample size with 122 and 724 cases, respectively [29-30]. Specificity reported in similar studies in the literature ranges from 74.9\% [30] to 98\% [17]. The diagnostic accuracy of FNA to detect malignancy for thyroid lesions is $85.88 \%$. Other studies reported $83.6 \%$ [29] to $89.46 \%$ [12] accuracy of FNA, which supports ours.

\section{Conclusions}

FNA cytologic examination of the thyroid nodule is a reliable diagnostic tool that carries high sensitivity and specificity. However, when non-surgical intervention is chosen in patients with benign FNA, the possibility of coexisting microcarcinoma with its variable prognosis should be taken into account and explained to the patient.

\section{Additional Information \\ Disclosures}

Human subjects: Consent was obtained by all participants in this study. King Fahad Medical City Institutional Review Board Committee issued approval H-01-R-012. Animal subjects: All authors have confirmed that this study did not involve animal subjects or tissue. Conflicts of interest: In compliance with the ICMJE uniform disclosure form, all authors declare the following: Payment/services info: All authors have declared that no financial support was received from any organization for the submitted work. Financial relationships: All authors have declared that they have no financial relationships at present or within the previous three years with any organizations that might have an interest in the submitted work. Other relationships: All authors have declared that there are no other relationships or activities that could appear to have influenced the submitted work.

\section{References}

1. Schlumberger M, Pacini F, Wiersinga WM, et al.: Follow-up and management of differentiated thyroid carcinoma: a European perspective in clinical practice. Eur J Endocrinol. 2004, 151:539-548. 10.1530/eje.0.1510539

2. Hegedüs L: The thyroid nodule. N Engl J Med. 2004, 351:1764-1771. 10.1056/NEJMcp031436

3. National Cancer Institute. SEER program. Cancer statistics review 1975-2016 . (2019). https://seer.cancer.gov/statfacts/html/thyro.html.

4. Hay ID, Klee GG: Thyroid cancer diagnosis and management. Clin Lab Med. 1993, 13:725-734. 10.1016/s0272-2712(18)30436-0

5. Teixeira G V, Chikota H, Teixeira T, Manfro G, Pai SI, Tufano RP: Incidence of malignancy in thyroid nodules determined to be follicular lesions of undetermined significance on fine-needle aspiration. World J Surg. 2012, 36:69-74. 10.1007/s00268-011-1336-8

6. Cibas ES, Ali SZ: The 2017 Bethesda system for reporting thyroid cytopathology . Thyroid. 2017, 27:13411346. 10.1089/thy.2017.0500

7. Haugen BR, Alexander EK, Bible KC, et al.: 2015 American Thyroid Association management guidelines for adult patients with thyroid nodules and differentiated thyroid cancer: the American Thyroid Association Guidelines Task Force on thyroid nodules and differentiated thyroid cancer. Thyroid. 2015, 26:1-133. 10.1089/thy.2015.0020

8. Hedinger C, Dillwyn Williams E, Sobin LH: The WHO histological classification of thyroid tumors: a commentary on the second edition. Cancer. 1989, 63:908-911. 10.1002/1097-0142(19890301)63:5<908::AIDCNCR2820630520>3.0.CO;2-I

9. Werga P, Wallin G, Skoog L, Hamberger B: Expanding role of fine-needle aspiration cytology in thyroid diagnosis and management. World J Surg. 2000, 24:907-912. 10.1007/s002680010163

10. Mistry SG, Mani N, Murthy P: Investigating the value of fine needle aspiration cytology in thyroid cancer . J Cytol. 2011, 28:185-190. 10.4103/0970-9371.86345

11. Sinna EA, Ezzat N: Diagnostic accuracy of fine needle aspiration cytology in thyroid lesions . J Egypt Natl Canc Inst. 2012, 24:63-70. 10.1016/j.jnci.2012.01.001

12. Machała E, Sopiński J, Iavorska I, Kołomecki K: Correlation of fine needle aspiration cytology of thyroid gland with histopathological results. Pol Przegl Chir. 2018, 90:1-5. 10.5604/01.3001.0012.4712

13. Lew JI, Snyder RA, Sanchez YM, Solorzano CC: Fine needle aspiration of the thyroid: correlation with final histopathology in a surgical series of 797 patients. J Am Coll Surg. 2011, 213:185-188. 
10.1016/j.jamcollsurg.2011.04.029

14. Her-Juing Wu H, Rose C, Elsheikh TM: The Bethesda system for reporting thyroid cytopathology: an experience of 1,382 cases in a community practice setting with the implication for risk of neoplasm and risk of malignancy. Diagn Cytopathol. 2012, 40:399-403. 10.1002/dc.21754

15. Al-Shraim MM, Kaood OM, Hussein MR, Al-Ahmary AM: Assessment of malignancy rate in thyroid nodules according to the Bethesda system of fine-needle aspiration. Report from a tertiary center in the Southwestern region of Saudi Arabia. Saudi Med J. 2012, 33:167-171.

16. Al-Rikabi AC, Al-Omran M, Cheema M, El-Khwsky F, Al-Nuaim A: Pattern of thyroid lesions and role of fine needle aspiration cytology (FNA) in the management of thyroid enlargement: a retrospective study from a teaching hospital in Riyadh. APMIS. 1998, 106:1069-1074. 10.1111/j.1699-0463.1998.tb00260.x

17. Caraway NP, Sneige N, Samaan NA: Diagnostic pitfalls in thyroid fine-needle aspiration: a review of 394 cases. Diagn Cytopathol. 1993, 9:345-350. 10.1002/dc.2840090320

18. Davies L, Welch HG: Increasing incidence of thyroid cancer in the United States, 1973-2002 . J Am Med Assoc. 2006, 295:2164-2167. 10.1001/jama.295.18.2164

19. Roti E, degli Uberti EC, Bondanelli M, Braverman LE: Thyroid papillary microcarcinoma: a descriptive and meta-analysis study. Eur J Endocrinol. 2008, 159:659-673. 10.1530/EJE-07-0896

20. Davies L, Welch HG: Current thyroid cancer trends in the United States . JAMA Otolaryngol Head Neck Surg. 2014, 140:317-322. 10.1001/jamaoto.2014.1

21. Noguchi S, Yamashita H, Uchino S, Watanabe S: Papillary microcarcinoma. World J Surg. 2008, 32:747-753. 10.1007/s00268-007-9453-0

22. Hsu YC, Lee JJ, Chien MN, Chen MJ, Leung CH, Cheng SP: Is papillary thyroid microcarcinoma a biologically different disease? A propensity score-matched analysis. J Surg Oncol. 2019, 120:1023-1030. 10.1002/jso.25670

23. Miyauchi A: Clinical trials of active surveillance of papillary microcarcinoma of the thyroid . World J Surg. 2016, 40:516-522. 10.1007/s00268-015-3392-y

24. Miyauchi A, Ito Y, Oda H: Insights into the management of papillary microcarcinoma of the thyroid . Thyroid. 2018, 28:23-31. 10.1089/thy.2017.0227

25. Ito Y, Miyauchi A, Oda H: Low-risk papillary microcarcinoma of the thyroid: a review of active surveillance trials. Eur J Surg Oncol. 2018, 44:307-315. 10.1016/j.ejso.2017.03.004

26. Musani MA, Khan FA, Malik S, Khambaty Y: Fine needle aspiration cytology: sensitivity and specificity in thyroid lesions. J Ayub Med Coll Abbottabad. 2011, 23:34-36.

27. Moon H-G, Jung E-J, Park S-T, et al.: Role of ultrasonography in predicting malignancy in patients with thyroid nodules. World J Surg. 2007, 31:1410-1416. 10.1007/s00268-007-9013-7

28. Dottorini ME, Mansi L: P. Perros (ed), British Thyroid Association, Royal College of Physicians. Guidelines for the management of thyroid cancer. Report of the Thyroid Cancer Guidelines Update Group. Eur J Nucl Med Mol Imaging. 2008, 35:1218-1219. 10.1007/s00259-008-0763-8

29. Borgohain R, Kumar Lal R, Chatterjee P, Brahma N, Khanna S: A study of cyto-histological correlation in the diagnosis of thyroid swelling. IOSR J Dent Med Sci. 2014, 13:46-49. 10.9790/0853-131144649

30. Sharma C: Diagnostic accuracy of fine needle aspiration cytology of thyroid and evaluation of discordant cases. J Egypt Natl Canc Inst. 2015, 27:147-153. 10.1016/j.jnci.2015.06.001 